\title{
The Seasonal Distribution of Macroplankton as shown by Catches in the 2-metre Stramin Ring-trawl in Off-shore Waters off Plymouth.
}

\author{
By \\ F. S. Russell, \\ Naturalist at the Plymouth Laboratory.
}

In past years during the study of the vertical distribution of macroplankton much information has been obtained on the abundance of the different animals in the catches at different times of the year. Results have already been published to show the seasonal distribution of pelagic young fish (4), and also on the abundance and composition of the Sagitta population $(5$, p. 565). In this latter study the change over from the predominance of Sagitta elegans to that of S. sctosa during the years 1930 to 1932 is striking. During the examination of catches it has been noticed that each year has been characterised at some time by the abundance of a species which may occur only in small numbers, if at all, in other years. This has been especially noticeable in the composition of the medusa population. It is necessary to follow these changes through a number of years to see if the presence of certain plankton organisms cannot be used as an index of conditions that may lead to other fluctuations such as those of the fisheries.

During 1930 and part of 1931 weekly catches of the 2-metre stramin ring-trawl were counted in order to obtain precise information on the seasonal abundance of the different animals throughout a whole year. The collections were made by oblique hauls of half an hour duration at the station 2 miles east of the Eddystone (4). Counts were made as on previous occasions by picking out directly distinctive and unusual animals and counting every animal in a $\mathrm{T}^{\frac{1}{0}}$ th sample of the remainder (2, p. 776).

The full results are given in the attached table which gives instructive information on the seasonal variation in abundance of the different animals, and their relative importance in numbers one to another. It seems unnecessary to analyse the tables further since any worker can abstract from it the data he may desire. One or two points must, however, be borne in mind.

1. The animals are all of a size that is normally retained in good condition by the stramin net. For instance, the medusæ are generally wellgrown individuals ; the earliest stages, while perhaps not small enough to pass through the meshes, are generally too badly damaged allow of 
definite identification. A useful indication of the size of disregarded medusæ is given by the absence of Rathkea octopunctata in Table I, which is common in tow-nettings in the earlier months of the year. This applies also to all other animal groups; the earliest stages of decapod larvæ do not appear in numbers in the catches as many will pass through the meshes.

In considering the seasonal distribution of any animal it must therefore be remembered that its first appearance in the catches does not imply the actual beginning of its abundance in the plankton. A period of two or three weeks should be allowed for the growth of the earliest stages to a size sufficient to appear in the catches.

2. The table does not contain any data on the abundance of young fishes which has been dealt with in another report (4).

3. In examining the data the vertical distribution of the various animals must be considered. The figures for Tomopteris helgolandica show, for instance, a great increase in July and August. This must only be regarded as an increase in abundance in the plankton; at other times the Tomopteris may actually be present in the area, but in the unsampled layers very near the bottom (see 3 , from which data can be obtained for most of the animals).

4. The names given in the table are those used in the Plymouth Marine Fauna (1931). Phialidium sp. will be mostly Phialidium hemispharicum : Phialella cymbaloides will also occur though not so commonly, as also a few Mitrocomclla brownei. It was not possible in the time to analyse all the Decapod larvæ into species : the species that comprise each group can be found in the Plymouth Marine Fauna, where also in many cases is given the time of year at which the larval stages are most prevalent. Pandalid larvæ must be taken to include all species contained under Pandalidæ, Hippolytidæ, and Processidæ. Mysid sp. in summer consist chiefly of young specimens.

5. Examination of catches over a number of years has shown that there are species which can normally be expected to appear regularly each year. It is felt that these results are a fairly reliable picture of the average ringtrawl plankton catches to be found throughout any year. The following species, however, appeared in exceptional numbers and cannot be regarded as characteristic, but only for the year in question at the times they appeared :-

Aglantha rosea, Liriope exigua, and Stephanomia bijuga : Clione limacina and adult Meganyctiphanes norvegica also appeared in unusual numbers at the beginning of the year, although the larvæ of the latter are usually present then.

The results are to form a basis with which future years can be compared. Marked differences have been noticed in other years and it is hoped to 
publish these results in a separate report when sufficient data have been gathered. It should then be possible to decide on certain species whose appearance in the plankton should be watched for in future years. It is especially desirable to see whether any correlation can be found between the appearance of one characteristic species and that of another. The desirability of such observations was already stressed many years ago when Allen (1) prepared a list of species for special observation. Now that regular collections of these larger plankton animals are obtainable throughout the year an opportunity is afforded to attempt this study over a period of a number of years.

\section{REFERENCES.}

1. Allen, E. J. The Distribution of Marine Plankton. Journ. Mar. Biol. Assoc., N.S., Vol. IV, No. 4, pp. 408-409, 1897.

2. Russell, F. S. The Vertical Distribution of Marine Macroplankton. An Observation on Diurnal Changes. Ibid., Vol. XIII, No. 4, p. $769,1925$.

3. The Vertical Distribution of Miarine Macroplankton. V. The Distribution of Animals caught in the Ring-trawl in the Daytime in the Plymouth Area. Ibid., Vol. XIV, No. 3, pp. 557-608, 1927.

4. - The Seasonal Abundance and Distribution of the Pelagic Young of Teleostean Fishès caught in the Ring-trawl in Offishore Waters in the Plymouth Area. Ibid., Vol. XVI, No. 3, pp. 707-722, 1930.

5. - On the Biology of Sagitta. IV. Observations on the Natural History of Sagitta elegans Verrill and Sagitta setosa J. Müller in the Plymouth Area. Ibid., Vol. XVIII, No. 2, pp. 559-574. 
TABLE I.

Numbers of Animals in Half-hour Oblique Hauls with the 2-metre Stramin Ring-trawl taken 2 miles East of the Eddystone.

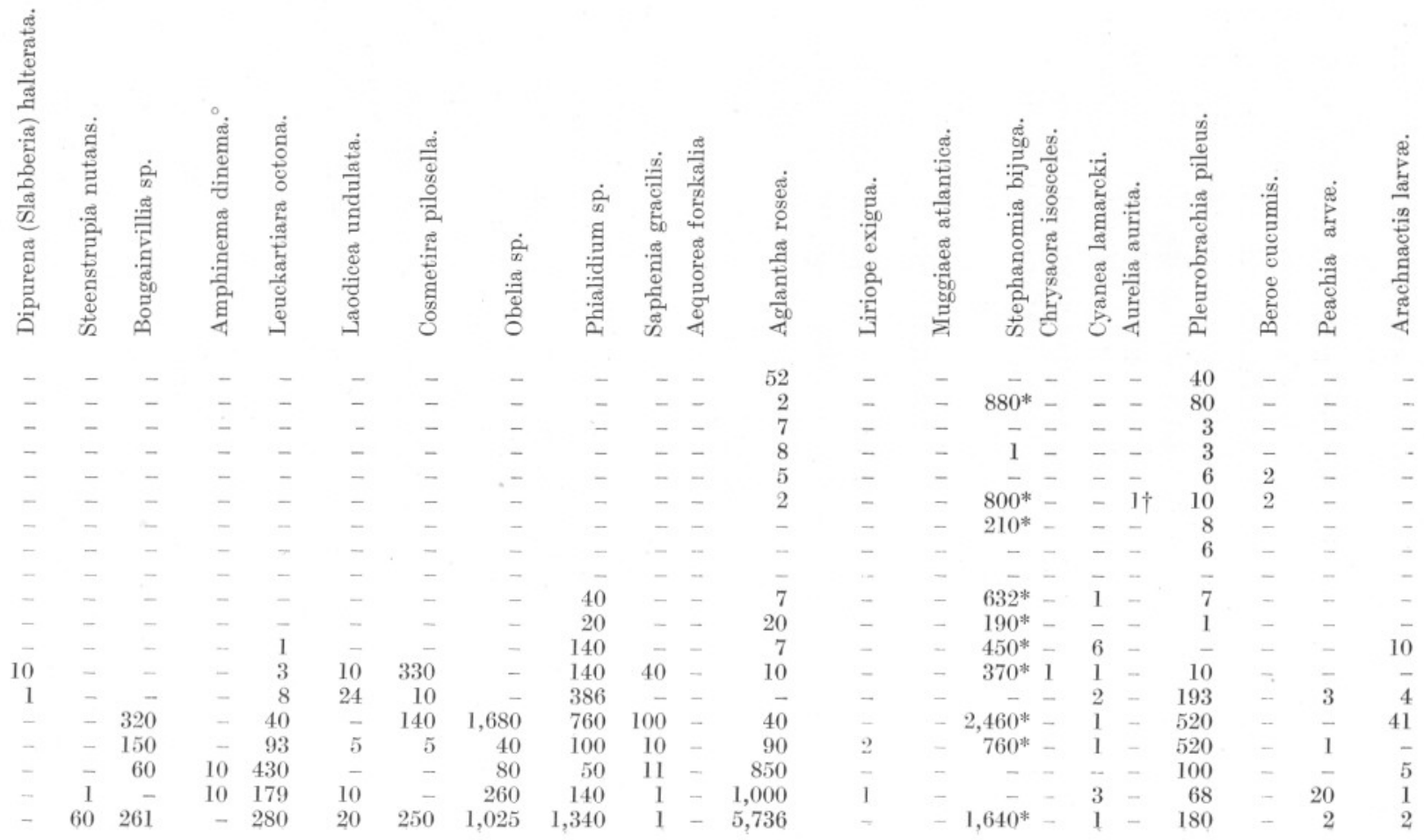




\begin{tabular}{|c|c|c|c|c|c|c|c|c|c|c|c|c|c|c|c|c|c|c|c|c|c|c|c|c|c|}
\hline July & 4th & . & . & - & - & 161 & - & 129 & - & 180 & 800 & 920 & - & - & 1,932 & - & - & - & - & - & - & 80 & 20 & 4 & $=$ \\
\hline, & 9th & . & . & - & 120 & - & - & 270 & - & 600 & 43,800 & 3,780 & 90 & - & 6,150 & 1 & - & 30 & - & - & - & - & 120 & 15 & - \\
\hline ,, & 14th & . & . & - & - & - & 1,110 & 180 & - & 180 & 24,540 & 2,970 & 90 & - & 11,040 & - & - & - & - & - & - & - & 30 & 94 & - \\
\hline , & 23rd & . & . & - & - & - & 330 & 180 & - & 1,530 & 1,080 & 5,790 & 150 & 1 & 28,440 & - & - & - & - & 2 & - & - & 21 & 127 & - \\
\hline & 29th & . & . & - & - & - & 990 & 60 & - & 870 & 810 & 3,750 & - & - & 23,040 & - & - & - & - & 3 & - & - & 39 & 35 & - \\
\hline Aug. & 7 th & . & . & - & - & - & - & 150 & 300 & - & 330 & 1,620 & - & 1 & 40,020 & - & - & - & - & - & - & - & 26 & - & - \\
\hline, & 14th & . & . & - & - & - & 1 & 44 & 120 & 60 & 160 & 4,020 & 40 & - & 33,220 & 446 & - & - & - & - & 1 & - & 137 & - & - \\
\hline , & 21st & . & . & - & 6 & - & - & 9 & 500 & - & - & 3,240 & 28 & - & 8,360 & 17,442 & 20 & $641^{*}$ & - & - & - & - & 33 & - & - \\
\hline, & 28th & . & . & - & - & - & - & $120:$ & 3,240 & - & - & 2,540 & 20 & - & 3,540 & 7,440 & 20 & $2^{*}$ & - & - & - & - & 32 & - & - \\
\hline Sept. & 3rd & . & . & - & 1 & - & - & 92 & 9,800 & - & 80 & 180 & 80 & - & 1,080 & 11,600 & 20 & $40^{*}$ & - & - & - & 1 & 8 & 17 & - \\
\hline & 11th & . & . & 20 & - & - & - & 21 & 4,160 & - & 100 & 4,300 & - & - & 340 & 4,280 & - & - & 1 & - & - & 161 & 33 & 55 & - \\
\hline , & 16th & . & . & - & - & - & - & 53 & 2,310 & 10 & 30 & 100 & - & - & 51 & 2,800 & 30 & - & - & - & - & 220 & 6 & 20 & - \\
\hline & 24th & . & . & - & - & - & - & 77 & 10 & - & - & 640 & - & - & 180 & 3,760 & 20 & - & - & - & - & 800 & 4 & - & - \\
\hline Oet. & 1st & . & . & - & - & - & - & 18 & - & - & - & 60 & - & - & 50 & 180 & - & - & - & - & - & 1,370 & 3 & - & - \\
\hline, & 7th & . & . & - & - & - & - & 3 & - & - & - & 340 & - & - & 20 & 660 & - & - & - & 1 & - & 500 & 4 & - & - \\
\hline ,, & 14th & & 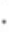 & - & - & - & - & - & - & - & 20 & 510 & - & - & - & 4,100 & 20 & - & - & - & - & - & - & - & - \\
\hline & 16th & $\cdot$ & • & - & - & - & - & - & - & - & 10 & 330 & - & - & - & 1,680 & - & - & - & - & - & - & - & - & - \\
\hline Nov. & 6th & . & & - & - & - & - & - & - & - & - & 20 & - & - & - & 1,650 & - & - & - & - & - & - & - & - & - \\
\hline & 13th & . & - & - & - & - & - & 10 & - & - & - & 20 & - & - & - & 980 & 50 & - & - & - & - & 20 & - & - & - \\
\hline & 20th & $\cdot$ & & - & - & - & - & - & - & - & - & 10 & - & - & 10 & 1,390 & 10 & - & - & - & - & - & - & - & - \\
\hline & 26th & . & - & - & - & - & - & - & - & - & - & 10 & - & - & - & 710 & 10 & - & - & - & - & - & - & - & - \\
\hline Dec. & 3rd & . & . & - & - & - & - & - & - & - & - & 100 & - & - & - & 1,115 & - & - & - & - & - & 10 & - & - & - \\
\hline , & 10th & . & . & - & - & - & - & - & - & - & - & 20 & - & - & - & 1,020 & 20 & - & - & - & - & 1 & - & - & - \\
\hline ,, & 17th & $\cdot$ & • & - & - & - & - & - & - & - & - & - & - & - & - & 690 & 50 & - & - & - & - & - & - & - & - \\
\hline ," & 22nd & . & . & - & - & - & - & - & - & - & - & - & - & - & - & 140 & 10 & - & - & - & - & - & - & - & - \\
\hline $\begin{array}{c}1931 \\
\text { Jan. }\end{array}$ & 1. & & & - & - & - & - & -7 & - & - & & & & & & 310 & 130 & - & - & - & - & - & & - & - \\
\hline , & 5 th & ${ }^{\circ}$ & . & 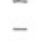 & - & - & - & - & - & - & - & $\overline{4}$ & - & - & - & $\begin{array}{l}510 \\
230\end{array}$ & 170 & - & - & - & - & - & - & - & - \\
\hline & 15th & . & . & - & - & - & - & - & - & - & - & 70 & - & - & - & 970 & 1,166 & - & - & - & - & - & - & - & - \\
\hline & 22nd & . & . & - & - & - & - & - & - & - & - & 30 & - & - & - & 104 & 130 & - & - & - & - & - & - & - & - \\
\hline & 26th & . & . & - & - & - & - & - & - & - & - & - & - & - & - & 10 & 10 & - & - & - & - & - & - & - & - \\
\hline Feb. & 6th & . & . & - & - & - & - & - & - & - & - & - & - & - & -- & - & 30 & - & - & - & - & - & - & - & - \\
\hline & 12th & . & . & - & - & - & - & - & - & - & - & 30 & - & - & - & - & - & - & - & - & $1 \dagger$ & - & - & - & - \\
\hline & 20th & . & . & - & - & - & - & 1 & - & - & - & 10 & - & - & - & - & 30 & - & - & - & - & - & - & - & - \\
\hline & 23rd & . & . & - & - & - & - & 20 & - & - & - & 80 & - & - & - & 2 & - & - & - & - & - & - & - & - & - \\
\hline † Mar. & .17th & . & . & - & - & - & - & 2 & - & - & - & 1,820 & - & - & - & - & - & - & - & 20 & - & - & - & - & - \\
\hline & 26th & . & . & - & - & - & - & 10 & 30 & - & 70 & 4,520 & - & - & - & - & 180 & - & - & 16 & - & - & - & - & - \\
\hline April & 1st & - & . & - & - & - & - & 2 & - & 20 & - & 220 & - & - & - & - & 20 & - & - & 3 & - & 20 & - & - & - \\
\hline, & 16th & ${ }^{\circ}$ & . & - & - & - & - & - & - & 660 & - & 12,120 & - & - & - & - & - & - & - & 23 & - & 40 & - & - & 1,480 \\
\hline
\end{tabular}


TABLE I-continued.

\begin{tabular}{|c|c|c|c|c|c|c|c|c|c|c|c|c|c|c|c|c|c|c|c|c|c|c|c|}
\hline & & & 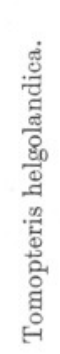 & 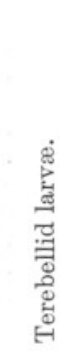 & 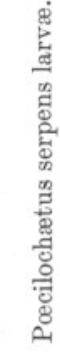 & 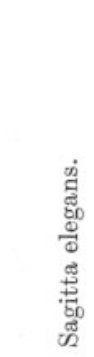 & 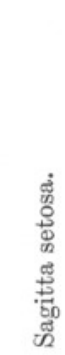 & 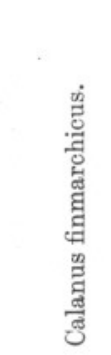 & 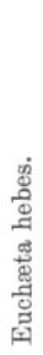 & 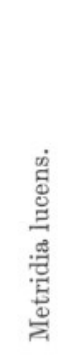 & 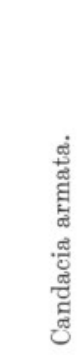 & 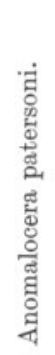 & 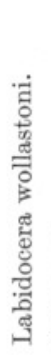 & 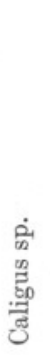 & 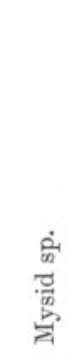 & 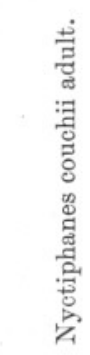 & 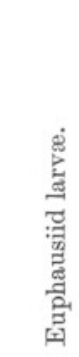 & 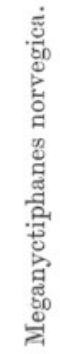 & 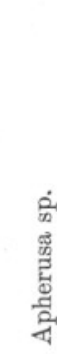 & 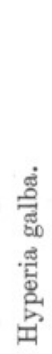 & 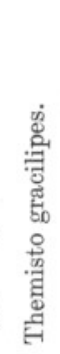 & 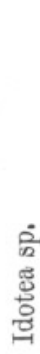 & 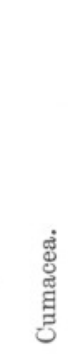 \\
\hline $\begin{array}{l}1930 \\
\text { Feb. }\end{array}$ & 4th & . & 140 & 2 & - & 11,360 & - & 1,200 & - & _- & 20 & - & - & - & 100 & 4,218 & - & 150 & - & - & 112 & - & \\
\hline, & 12 th & . & 770 & - & - & 16,270 & 130 & 540 & - & - & - & - & - & 1 & - & 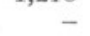 & 380 & - & - & - & - & - & - \\
\hline , & 19th & . & 251 & - & - & 10,411 & 389 & 1,280 & - & - & 60 & - & - & 1 & - & 20 & - & - & - & - & 1 & - & - \\
\hline & 26th & . & 3 & - & - & 452 & 110 & 580 & - & - & - & - & - & - & - & - & - & - & - & - & - & - & - \\
\hline Mar. & 5 th & . & 165 & - & - & 1,517 & 93 & 450 & - & - & - & - & - & - & - & 4 & - & - & - & - & 1 & - & - \\
\hline ,, & 12th & . & 33 & - & - & 455 & 5 & 260 & - & - & - & - & - & - & - & - & - & - & - & - & - & - & - \\
\hline ,, & 19th & . & - & - & - & 8 & - & - & - & - & - & - & - & 1 & - & - & - & - & - & - & - & - & - \\
\hline , & 27th & . & 3 & - & - & 1,860 & - & 420 & - & - & - & - & - & 2 & - & 2 & - & - & - & - & - & - & .. \\
\hline April & 2nd & . & - & - & - & 24 & 1 & 10 & - & - & - & 38 & - & 1 & - & - & - & - & - & - & 1 & - & - \\
\hline , & 11th & . & 3 & - & - & 240 & - & 440 & - & 60 & - & - & - & 1 & - & 2 & - & - & - & - & - & - & - \\
\hline , & 16th & . & - & - & - & 21 & - & 16,890 & - & 70 & 60 & 30 & - & 1 & - & 1 & 310 & 1 & - & - & - & - & - \\
\hline , & 24 th & . & - & - & 10 & 860 & - & 7,630 & - & - & 510 & - & - & 1 & - & - & 1,140 & - & - & - & 130 & - & - \\
\hline & 29 th & . & - & - & - & 39 & 1 & 960 & - & 60 & 80 & - & - & - & - & 1 & 540 & - & - & - & 2 & - & - \\
\hline May & 7 th & . & 7 & 386 & - & 77 & 2 & 318,450 & - & 193 & 772 & - & - & - & - & 2 & 2,702 & - & - & - & - & 3 & - \\
\hline, & 15 th & . & 10 & 60 & - & 243 & 7 & 24,720 & - & 80 & 260 & - & - & - & - & - & 580 & - & - & - & - & - & - \\
\hline, & 22nd & . & 10 & - & - & 101 & - & 11,210 & - & - & 50 & 20 & - & 1 & - & - & 450 & - & - & - & - & - & - \\
\hline June & 10th & . & 63 & - & 110 & 1,324 & - & 1,968 & - & - & 200 & 4 & - & 1 & 1 & 4 & 40 & - & - & - & - & - & - \\
\hline ,, & 19th & . & 25 & - & - & 637 & 6 & 6,592 & - & - & 220 & 22 & - & 2 & - & - & 20 & - & - & 5 & - & - & -- \\
\hline ,", & 26 th & . & 95 & - & - & 760 & - & 3,560 & - & 40 & 320 & 13 & - & 2 & 2 & - & 40 & - & 20 & - & 20 & - & - \\
\hline July & 4th & . & 10 & - & - & 2,218 & - & 2,023 & - & - & 200 & 62 & - & 2 & 2 & - & - & - & 20 & - & 1 & - & - \\
\hline & 9 th & . & 664 & - & - & 4,377 & 8 & 6,132 & - & - & 2,160 & 60 & - & 2 & 360 & - & - & - & 60 & - & 1 & - & - \\
\hline ", & 14 th & . & 741 & - & - & 3,942 & 4 & 8,370 & - & - & 930 & + & - & - & 152 & - & 30 & - & - & - & - & - & - \\
\hline ," & 23rd & . & 759 & - & 30 & 2,142 & 10 & 2,910 & - & 30 & 1,860 & 10 & - & 2 & 60 & 8 & 30 & - & 750 & - & - & -. & - \\
\hline , & 29 th & . & 376 & - & - & 1,423 & 12 & 1,007 & - & 30 & 270 & - & - & 4 & 6 & - & - & - & 240 & - & 1 & - & - \\
\hline
\end{tabular}




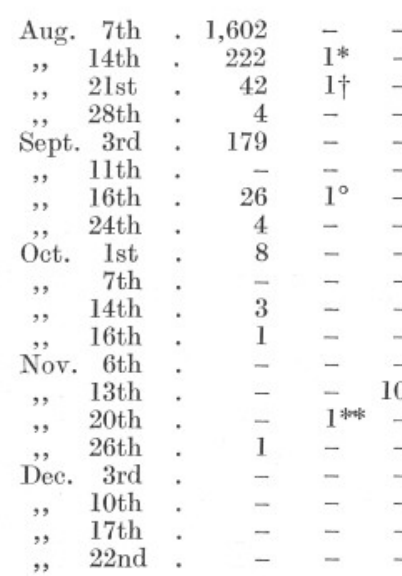

$\begin{array}{rr}7,800 & 36 \\ 5,210 & 5 \\ 2,038 & - \\ 1,018 & - \\ 1,222 & 47 \| \\ 4,398 & 760 \\ 558 & 46 \\ 270 & 87 \\ 102 & 45 \\ 74 & 369 \\ 303 & 683 \\ 229 & 258 \\ 441 & 300 \\ 159 & 120 \\ 32 & 73 \\ 61 & 84 \\ 730 & 1,080 \\ 360 & 162 \\ 262 & 372 \\ 72 & 78\end{array}$

$\begin{array}{rr}2,730 & - \\ 1,041 & - \\ 803 & - \\ 402 & - \\ 363 & - \\ 612 & - \\ 233 & - \\ 35 & - \\ 70 & - \\ 263 & - \\ - & - \\ 118 & - \\ - & - \\ 99 & - \\ 7 & - \\ 14 & - \\ 107 & - \\ 129 & - \\ 169 & - \\ 98 & -\end{array}$

$\begin{array}{lrrrr}- & 450 & - & - & 5 \\ - & 220 & 1 & - & 8 \\ - & 200 & - & - & 4 \\ - & 80 & - & - & 1 \\ - & 140 & 1 & - & - \\ - & 560 & 3 & - & 7 \\ - & 40 & 1 & 10 & - \\ - & - & 53 & - & - \\ - & 20 & - & - & 1 \\ - & 30 & - & - & 1 \\ - & 150 & - & - & 2 \\ - & 10 & 10 & - & - \\ - & 10 & - & - & 1 \\ - & 40 & - & - & - \\ - & - & - & - & 1 \\ - & 20 & - & - & - \\ - & 40 & - & - & 1 \\ - & 100 & - & - & 1 \\ - & 70 & - & - & - \\ - & 90 & - & - & -\end{array}$

$\begin{array}{rrrr}94 & 102 & - & - \\ 17 & - & 140 & - \\ - & - & 20 & - \\ - & - & - & - \\ 20 & - & - & - \\ - & 1 & 20 & - \\ - & - & - & - \\ - & - & 7 & - \\ - & & - & - \\ - & - & - & - \\ 1 & - & - & - \\ - & - & - & - \\ 1 & - & - & - \\ - & 1 & 10 & - \\ - & - & - & - \\ - & - & - & - \\ - & - & - & - \\ - & - & 121 & - \\ - & - & 30 & - \\ - & - & 10 & -\end{array}$

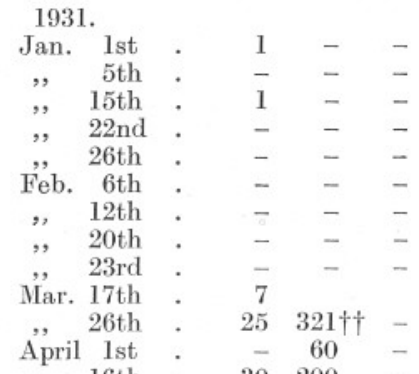

$\begin{array}{rr}210 & 249 \\ 109 & 59 \\ 1,765 & 339 \\ 172 & 270 \\ 117 & 101 \\ 51 & 2 \\ 83 & 17 \\ 816 & 239 \\ 474 & 134 \\ 7,502 & 221 \\ 491 & 333 \\ 935 & 1,048 \\ 764 & 385\end{array}$

$\begin{array}{rrrrrrr}20 & - & - & 60 & - & - & 2 \\ 80 & 10 & - & 120 & - & - & 1 \\ 190 & - & - & 150 & - & - & - \\ 56 & - & - & 20 & - & - & 1 \\ 19 & 10 & - & 20 & - & - & - \\ - & - & - & 20 & - & - & - \\ 40 & - & - & 10 & - & - & 1 \\ 446 & - & - & 220 & - & - & 1 \\ 343 & - & - & 420 & - & - & 1 \\ 110 & - & - & 50 & - & - & 3 \\ 320 & - & - & 220 & - & - & - \\ 1,260 & - & - & 180 & - & - & 1\end{array}$

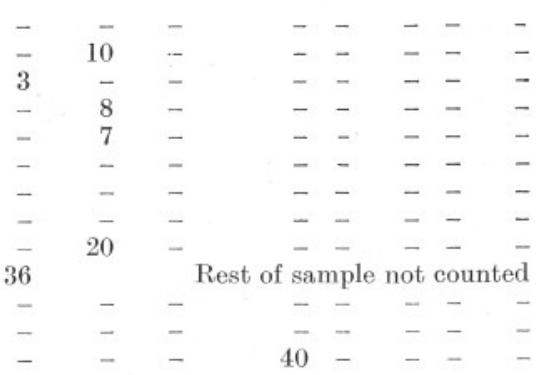

* Young Syllid. † Glycera. †† 1 Autolytus. 
TABLE I-continued.
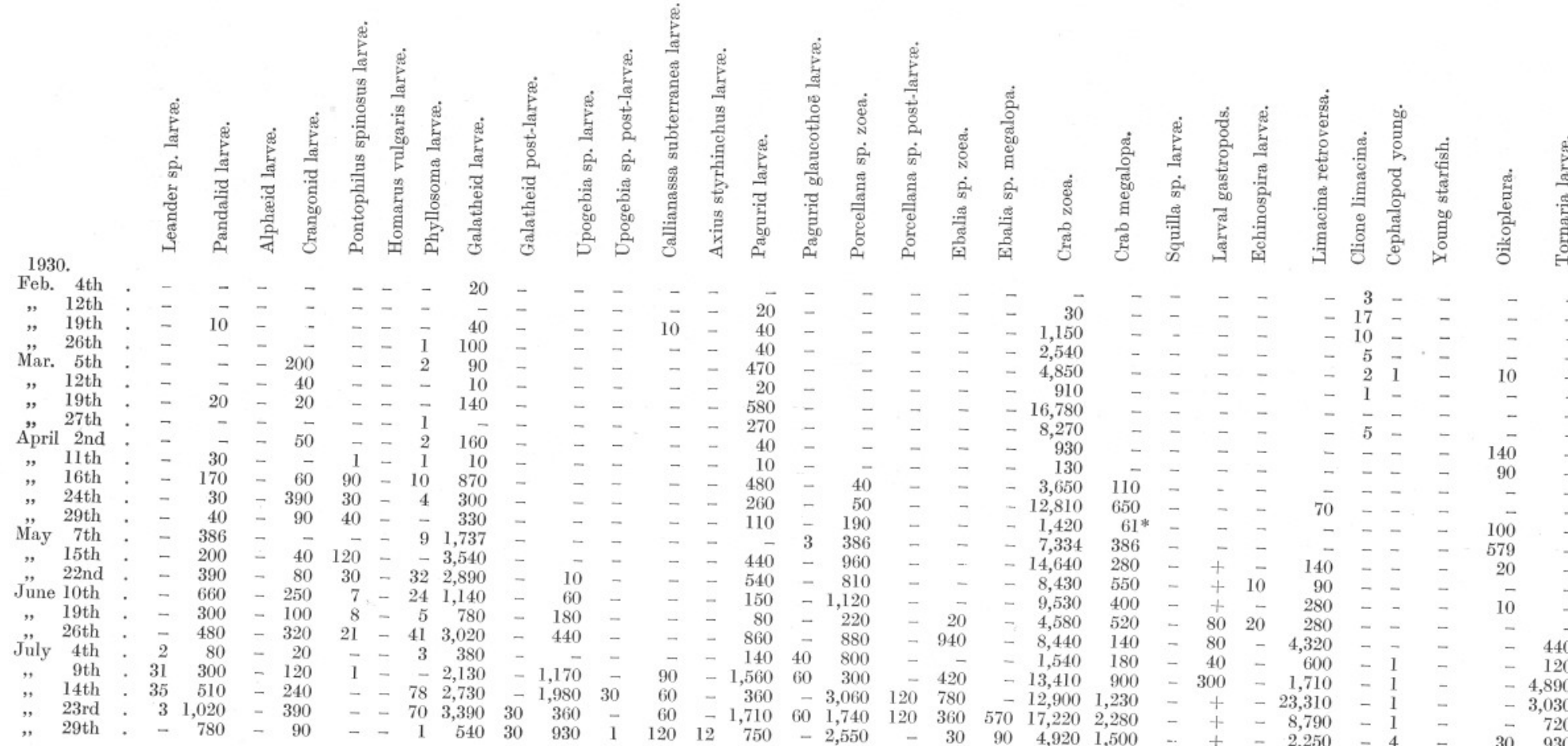
$20 \quad 17,787$
$110 \quad 13,792$
$4,020 \quad 11,888$
$2,500 \quad 4,041$
$5,620 \quad 23,17$
$2,750 \quad 13,799$
$3,850 \quad 26,945$
$800 \quad 26,419$
$900 \quad 5,888$
$1,737 \quad 335,784$
$860 \quad 53,302$
$190 \quad 2$ ry
$300 \quad 16,696$
$960 \quad 3$
$1,120 \quad 13,830$

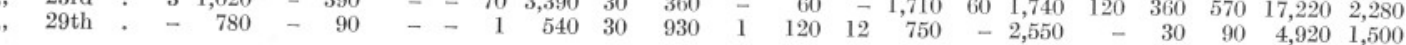

\begin{tabular}{l}
$-\quad 720$ \\
\hline 0
\end{tabular} 

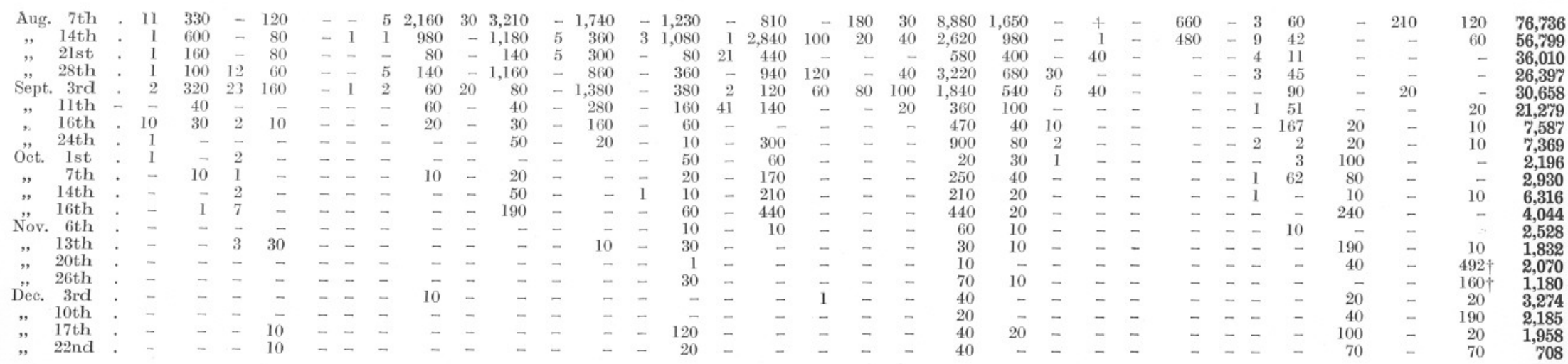

1931.

$$
\begin{aligned}
& \text { Jan. 1st } \\
& \text { " } \quad \text { 5th } \\
& \text { 22nd } \\
& \text { Feb. 6th } \\
& 12 \mathrm{th} \\
& 20 \text { th } \\
& \text { 23rd } \\
& \text { April 1st }
\end{aligned}
$$

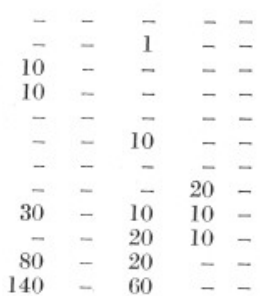
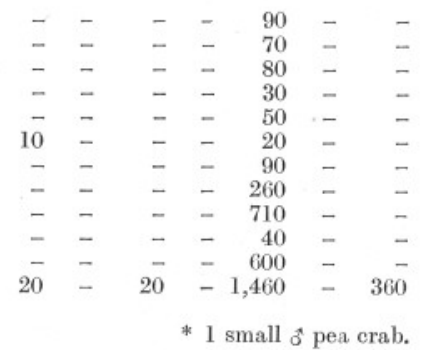

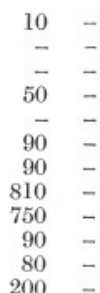
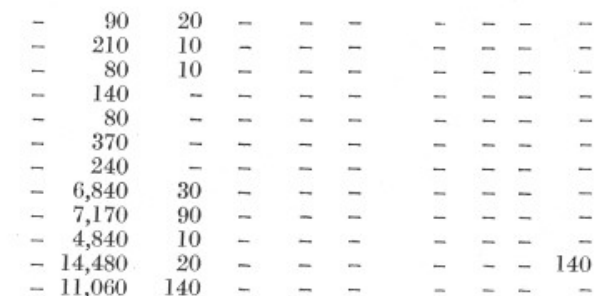

$\begin{array}{rr}60 & - \\ 40 & - \\ 590 & - \\ 110 & - \\ 70 & - \\ 50 & - \\ 50 & - \\ 110 & - \\ - & - \\ - & - \\ - & - \\ - & -\end{array}$

$\begin{array}{rr}90 & \mathbf{1 , 3 4 2} \\ 100 & \mathbf{1 , 2 2 0} \\ 1,270 & \mathbf{6 , 7 0 4} \\ 1,300 & \mathbf{2 , 4 3 1} \\ 640 & \mathbf{1 , 1 3 4} \\ 910 & \mathbf{1 , 5 6 3} \\ 530 & \mathbf{1 , 1 8 2} \\ 7,140 & \mathbf{1 6 , 9 7 3} \\ 7,090 & \mathbf{1 7 7 , 3 5 4} \\ 780 & \mathbf{1 1 , 9 4 9} \\ 80 & \mathbf{1 8 , 3 6 8} \\ 1,040 & \mathbf{3 1 , 6 8 3}\end{array}$


